\title{
Method of image quality assessment based on region of interest and Structural Similarity
}

\author{
Dai Li ${ }^{1, a}$, Tao Cheng ${ }^{2, b^{*}}$ \\ ${ }^{1}$ The College of Mechatronics and Control Engineering, Shenzhen University, Shenzhen, P. R. \\ China, 518060 \\ ${ }^{2}$ The College of Mechatronics and Control Engineering (The College of Urban Rail Transit), \\ Shenzhen University, Shenzhen, P. R. China, 518060 \\ alz_lidai@sina.com, ${ }^{\mathrm{b}}$ chengtao@szu.edu.cn
}

\begin{abstract}
Keywords: Structure Similarity(SSIM); image quality assessment; weighted coefficient ; Region-Of-Interest(ROI).

Abstract. In this paper, a method of image quality assessment based on visual regions of interest and Structural Similarity(SSIM) is proposed through combining human visual interest regions and structural similarity. In this algorithm, we firstly use the SSIM to separately assess those interest regions, and get weight of different regions through analyzing the influencing factors such as brightness, gray level, area size, and then work out the image quality of ROI by adding up those weights of different regions. Secondly we can get the assessment of the entire image by using the SSIM to evaluate the entire image. The final assessment of image is the result of adding up different weights of the entire image and ROI. What's more, the experiment results prove the method is more accurate and efficient than SSIM.
\end{abstract}

\section{Introduction}

With the development of image processing technology, it has great significance that how to more effectively assess an image, so as to provide reference for adjusting the image quality, optimizing algorithm and detection system. At present, there are two image assessment methods: subjective and objective. The subjective assessment regards subjective visual as the assessment criterion and depends on the observer to assess image quality; The objective assessment is a quantitative description of image quality by means of mathematical model, statistical analysis and so on. According to the level of dependence on the original image, the objective assessment is divided into three categories: full reference, weak reference and No-Reference. (1)Full Reference QA, assess image though the method of compare the original and evaluated image; (2) Weak reference QA ,it use some characteristic information obtained from the reference image to evaluate image quality; (3) No- reference QA ${ }^{[1]}$, it evaluates the image with no reference.

In the two methods, the subjective assessment needs special training for the personnel, and its process is time-consuming and money-wasting. Moreover, it cannot be used in real-time assessment because of it depends on the man's subjective vision. The full reference and weak reference assessment all depends on the reference image, while in some applications, the original image is often difficult or even impossible to achieve. Therefore, it weighs more important to study the No-Reference image quality assessment.

In the No-Reference quality assessment system, most of methods aim at the whole image, without considering observers quality requirements in different regions, so that some image assessment result do not match the subjective assessment. This essay is based on Structural Similarity definition algorithm and proposes an assessment method comprehensively considering the quality of whole image and Region-Of-Interest. Experimental results show that this method is consistent with subjective assessment results not only for the quality of whole image but also for partly-blur image ${ }^{[2]}$. 


\section{Method of image quality assessment based on Region-Of-Interest}

Due to the human physiological characteristics, people can only observe seriously one region of a picture and cannot identify other areas. Some research shows that when people observe a thing seriously, the visual effective area is only coin size. Meantime, human visual system has local contrast sensitivity that vision is only interested in a region of significant changes in the visual field. So people in the image observation will form a "Region-Of-Interest" ${ }^{\text {[3] }}$.

The method of image quality assessment based on Region-Of-Interest combines the human eye selectivity and the objective assessment method, which takes full consideration on visual characteristics in order to make the assessment result conform to subjective feeling.

Literature [4] introduced an improvement of the Mean Square Error (MSE) and Peak Signal-to-Noise Ratio (PSNR) used to evaluate the Region-Of-Interest.

$$
\begin{aligned}
& H M S E=\frac{\sum_{i=1}^{N} p_{i} \sum_{A_{i}}\left[f(m, n)-f^{\prime}(m, n)\right]^{2}+p_{i}^{\prime} \sum_{A_{L}}\left[f(m, n)-f^{\prime}(m, n)\right]^{2}}{\sum_{A-A_{l}}\left[f(m, n)-f^{\prime}(m, n)\right]^{2}} . \\
& H P S N R=10 \lg \frac{256 \times 256}{H M S E} .
\end{aligned}
$$

Notes: $A_{i}(i=1,2 \ldots \mathrm{N})$ stands for the Region-Of-Interest. $S_{i}(i=1,2 \ldots, \mathrm{N})$ is the area size of $A_{i} ; A_{l}$ is the rest area. $S$ means the total area of the image. The weighted coefficient of the interested is $p_{i}(i=1,2 \ldots$ $\mathrm{N}$ ); the weighted coefficient of the non-interested region is $p_{l}$.

\section{Reblur Theory}

In the image frequency domain analysis, it can be known that the image clarity has nothing to do with the low frequency component, decided only by the middle or high frequency component of the energy. Therefore, when the middle or high frequency components are filtered by a low pass filter, the image will become blurred. For a fuzzy image, the middle or high frequency components of the image are relatively small, so a low pass filter has little effect on the image quality. For the clear image, the low pass filter will make it lose the middle or high frequency component, thus the image quality will be poor. Based on this, the change of adjacent pixel values of two images before and after filtering can be compared to measure the clarity ${ }^{[5,6]}$.

\section{Structural Similarities}

The reference[7-10] proposed that the structural distortion is the key factor in the image quality assessment, and pointed out the Structural Similarity theory and Structural Similarity Index Measurement(SSIM). SSIM is divided the image into three parts:lightness, contract and structure. They are separately compared, then the weighted product to work out the SSIM value.

$$
\begin{aligned}
& \operatorname{SSIM}(x, y)=[l(x, y)]^{\alpha}[c(x, y)]^{\beta}[s(x, y)]^{\gamma} . \\
& \left\{\begin{array}{l}
l(x, y)=\frac{2 u_{x} u_{y}+c_{1}}{u_{x}^{2}+u_{y}^{2}+c_{1}} \\
c(x, y)=\frac{2 \sigma_{x} \sigma_{y}+c_{2}}{\sigma_{x}^{2}+\sigma_{y}^{2}+c_{2}}
\end{array}\right. \\
& s(x, y)=\frac{\sigma_{x y}+c_{3}}{\sigma_{x} \sigma_{y}+c_{3}}
\end{aligned}
$$


Note: $\mathrm{u}_{\mathrm{x}}, \mathrm{u}_{\mathrm{y}}$ as the average lightness of the reference image and evaluated image, $\sigma_{\mathrm{x}}, \sigma_{\mathrm{y}}$, as standard deviation, $\sigma_{\mathrm{xy}}$ is the covariance. $c_{1}, c_{2}, c_{3}$ as the constant. When $\alpha=\beta=\gamma, c_{3}=c_{2} / 2$, Eq. (4) can be simplified as:

$$
S S I M=\frac{\left(2 u_{x} u_{y}+c_{1}\right)\left(2 \sigma_{x y}+c_{2}\right)}{\left(u_{x}^{2}+u_{y}^{2}+c_{1}\right)\left(\sigma_{x}^{2}+\sigma_{y}^{2}+c_{2}\right)}
$$

SSIM has better effect on static image quality assessment, but for overall badly blurred image, the local blur quality but wholly acceptable image, and local blur image, the effect is not ideal but very inconsistent with subjective feeling ${ }^{[8]}$.

\section{Method of image quality assessment based on Region-Of-Interest and Structural Similarity}

The assessment method is shown in Fig.1:

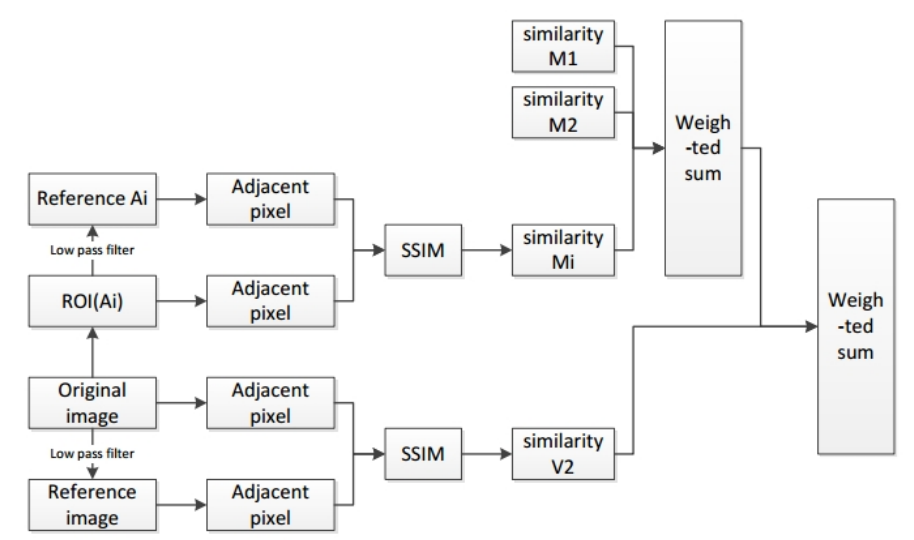

Fig.1 the steps of assessment method

In view of the reblur theory and Structural Similarity principle, this paper proposed a method of image assessment based on the Structural Similarity of the visual interest region by considering the overall quality of the image and the visual characteristics of the human eye. The assessment method is divided into two parts: the Region-Of-Interest and the overall image assessment. The specific process is as follows:

ROI(Region-Of-Interest) assessment. ROI assessment is the assessment of the possible interest regions in the image. Assume that the image has $\mathrm{N}$ regions $A_{i}(i=1,2 \ldots N)$, the area is $S_{i}(i=1,2 \ldots \mathrm{N})$. By low pass filtering $A_{i}$, the reference image of $A_{i}$ is obtained. Then compare the Structural Similarity $M_{i}$ of the two structures and weight sum of $M_{i}$, Finally the weighted coefficient $p_{i}$ is determined by the intensity, area size and gray level:

(1) The influence of brightness on interest

$$
s_{i}=k\left|\lg \frac{I_{i}}{I_{o i}}\right| .
$$

Note: $I_{i}$ is the brightness of ROI area $A_{i}, I_{o i}$ is background brightness, $s_{i}$ is the weighted coefficient caused by brightness;

(2) The influence of area on interest

$$
t_{i}=j \lg \frac{\sqrt{s}}{\sqrt{s-s_{i}}} .
$$


Note: $s$ is the area of the full image, $s_{i}$ is the area of the ROI, $t_{i}$ is the weighted coefficient caused by area;

(3) The influence of gray on interest

$$
\omega_{i}=h \lg \frac{r_{i}}{r_{o i}}
$$

Note: $r_{i}$ is the average gray of the boundary of ROI, $r_{o i}$ is the average gray of the adjacent outside the boundary, $\omega_{i}$ is the weighted coefficient caused by gray;

Weight $p_{i}$ :

$$
p_{i}=\sqrt{s_{i}^{2}+t_{i}^{2}+\omega_{i}^{2}} \text {. }
$$

(4) The assessment of ROI area $V_{1}$ :

$$
V_{1}=\sum_{i=1}^{N} p_{i} \times M_{i}
$$

Whole image assessment. Whole image assessment is a comprehensive assessment of the whole image. First by low-pass filtering of the evaluated image to calculate the image structure similarity $V_{2}$, and weighting sum the value $V_{l}$ and the full image assessment value $V_{2}$, the comprehensive assessment of the evaluated image will appear.

$$
\begin{aligned}
& \text { blur }=\alpha \times V_{1}+\beta \times V_{2} . \\
& \text { RSSIM }=1-\text { blur } .
\end{aligned}
$$

Experimental verification. This paper adopts the Release Database 2 image database of University of Texas-Austin ${ }^{[11]}$ to verify the assessment method. From the image database, we choose a set of images with different blur degree. Then respectively use a simple SSIM assessment method and the assessment method based on the Structural Similarity of the interest region, the part assessment results are shown in Figure 1. Experimental results from comparing results of the two assessment methods and the blur degree relationship to the image provided in the image database are shown in table 1.

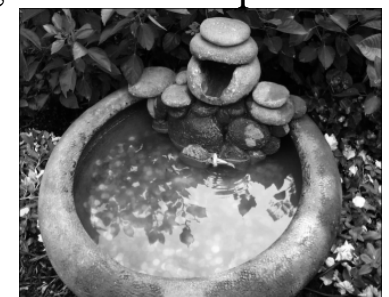

Fig.a

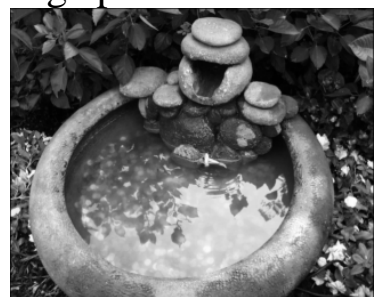

Fig.b

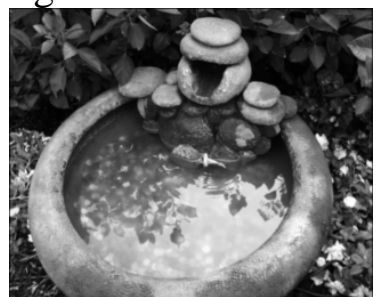

Fig. c

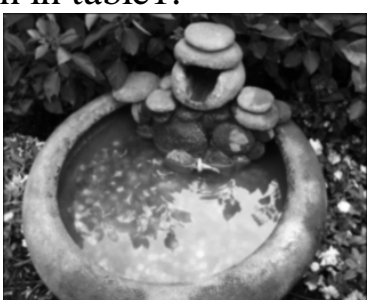

Fig. d

Table1 the experimental data

\begin{tabular}{c|c|c|c|c|c|c}
\hline Image & $\mathrm{a}$ & $\mathrm{b}$ & $\mathrm{c}$ & $\mathrm{d}$ & $\mathrm{e}$ & $\mathrm{f}$ \\
\hline blur & 0 & 0.562467 & 0.848926 & 1.450490 & 1.708303 & 2.510388 \\
\hline SSIM & 0.0829 & 0.054 & 0.0324 & 0.0118 & 0.081 & 0.031 \\
\hline RSSIM & 0.4442 & 0.4262 & 0.4126 & 0.4001 & 0.3979 & 0.3951 \\
\hline
\end{tabular}




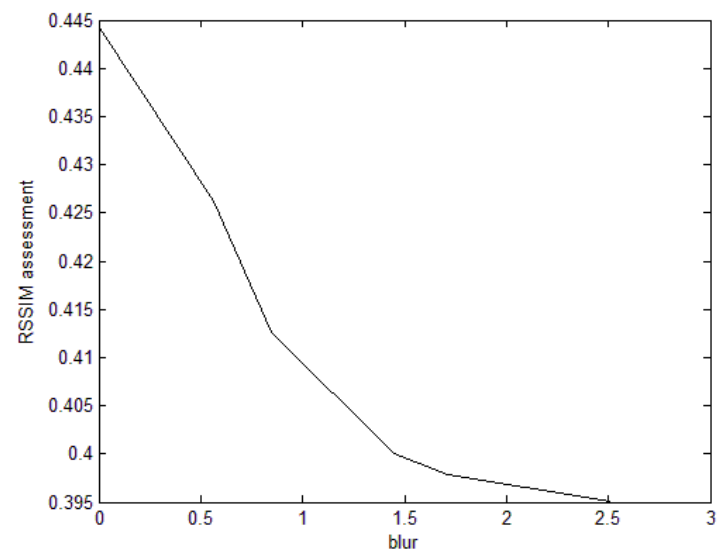

Fig.2 RSSIM assessment

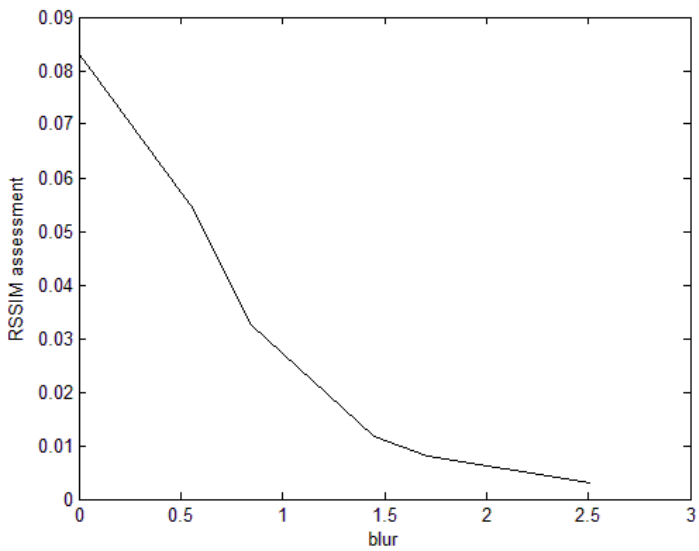

Fig.3 SSIM assessment

Figure 2 and Figure 3 show the relationship between SSIM and RSSIM and the gived blur image in the image database. From the table 1 and figures. The effect of RSSIM image quality assessment method and SSIM assessment are not big difference. In a low degree of blur, the sensivity of the RSSIM is higher than SSIM, while in the higher part, RSSIM sensitivity is lower. In somehow, it is better to assess the serious blur image.

In order to verification the effect for local blur but whole acceptable images though the method of image quality assessment based on Region-Of-Interest and Structural Similarity (RSSIM). This paper use the Gaussian blur to filter a small region of the original image. Some different degrees of blur image are partly shown in Figure followed. Then, the local blur image is evaluated by SSIM and RSSIM respectively, and the assessment value of the two are compared. Experimental data are shown in table 2.

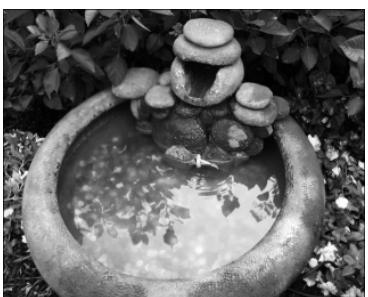

Fig.e

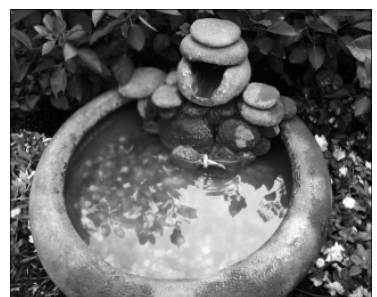

Fig.f

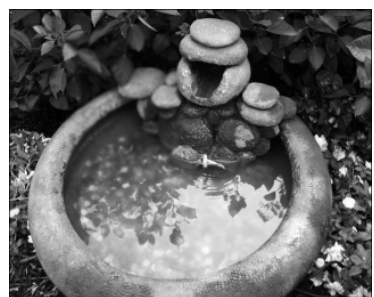

Fig.g

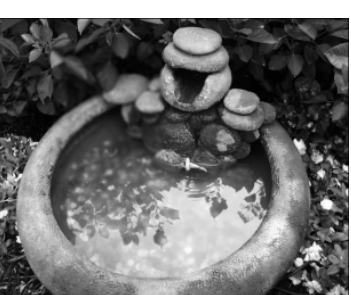

Fig.h

Table2 the part blur experiment data

\begin{tabular}{c|c|c|c|c|c|c}
\hline image & $\mathrm{e}$ & $\mathrm{f}$ & $\mathrm{g}$ & $\mathrm{h}$ & $\mathrm{i}$ & $\mathrm{j}$ \\
\hline blur & $3 \times 3$ & $4 \times 4$ & $5 \times 5$ & $6 \times 6$ & $7 \times 7$ & $8 \times 8$ \\
\hline SSIM & 0.0717 & 0.0703 & 0.0695 & 0.0697 & 0.0690 & 0.0690 \\
\hline RSSIM & 0.4293 & 0.4285 & 0.4281 & 0.4279 & 0.4278 & 0.4277 \\
\hline
\end{tabular}

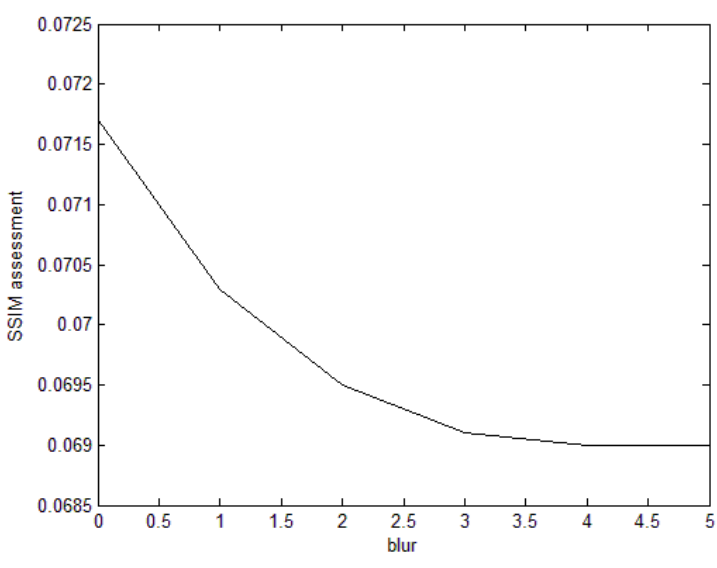

Figure4. SSIM assessment for locality blur

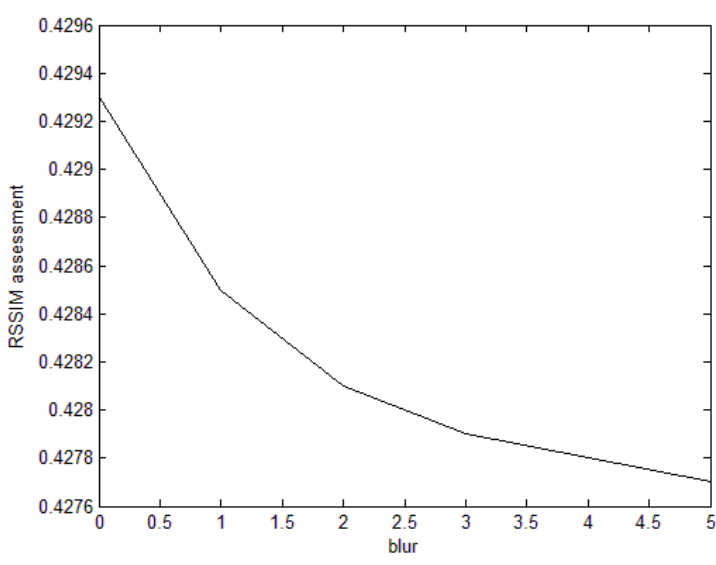

Figure5. RSSIM assessment for locality blur 
From figure 4 and figure 5 of the whole acceptable but local blur image assessment, RSSIM has a better linearity compared with SSIM. Meantime in badly-blur images, SSIM assessment value has little change with the difference of blur, while RSSIM is still maintained a good assessment.

\section{Conclusion}

In this paper, a method of image quality assessment based on Region-Of-Interest and Structural Similarity is proposed. The experimental results show that the method has better linearity than the pure SSIM assessment method. At the same time in the badly-blur image and the local blur but whole acceptable image, RSSIM assessment method shows its advantage. However, this paper simply selects the full image weighted coefficient with 0.4, the image of interest area with 0.6 in the weight sum of Structural Similarity based on interest region and the overall similarity value, which is to highlight the importance of the full quality of the image. How to choose the weight to better in line with human subjective vision and linear needs further study.

\section{Acknowledgements}

This work was financially supported by

the National Natural Science Foundation of China (11274336), Special Funds Project of Public

Welfare Research \& Capacity Building in Guangdong Province in 2014: Research \& Development of IoT-based Intelligent Ubiquitous-Perceiving Key Technology for Manufacturing Execution System (Grant No. 2014A010103034).

\section{References}

[1] ONG E, LIN W, LU Z, et al, No-Reference quality metric for measuring image blur, IEEE International Conference on Image Processing. 34(2003)467-472.

[2] Li Z L,Li X H, Ma L L, et al, Research of Definition Assessment based on No reference Digital Image Quality, Remote Sensing Technology and Application. 26(2011)239-246.

[3] LIU J Q,MA L,XIANG Y, et al, Image Quality Assessment of Dual-scale Structure Similarity Based on Region-Of-Interest, computer engineering. 40(2014)232-235.

[4] LU X G,WANG Y F,HU W G, et al, A Method of Image Quality Assessment Based on Visual Regions of Interest, Micro computer information. 21(2005)95-97.

[5] Crete F, Dolm iere T, Ladret P, et al, The Blur Effect: Perception and Estimation with a New No-Reference Perceptual Blur Metric [C]//Proceeding of the SPIE. San Jose, 29 January 2007,649201.11.

[6] FAN Y Y, SHEN X H, SANG Y J, No reference image sharpness assessment based on contrast sensitivity [J].Optics and Precision Engineering. 19(2011)2485-2493.

[7] Yang C L, Chen G H, Xie S L, Gradient Information based Image Quality Assessment, Chinese Journal of Electronics. 35(2007)1313-1317.

[8] Xie X F, Zhou J, Wu Q Z, No-Reference Quality Index for Image Blur, Computer Application. 30(2010)921-924.

[9] Wang Z, Bovik A C, Sheik H R, et al, Image Quality Assessment: from Error Visibility to Structural Similarity, IEEE Transactions on Image Processing. 13(2004)600-612.

[10] WANG Y Q. LIU W Y, WANG Y, Image quality assessment based on local variance and structure similarity [J].Journal of Optoelectronics • Laser. 19(2008)1546-1553.

[11] Sheikh H R, Wang Z, Cormack L, et al, LIVE Image Quality Assessment Database Release 2 [DB/OL]. 